\title{
Juicio y ser. Kant y Fichte en la encrucijada hacia el romanticismo
}

Mònica Carbó Ribugent

\section{(2) OpenEdition}

Journals

Edición electrónica

URL: http://journals.openedition.org/ref/425

DOI: $10.4000 /$ ref.425

ISSN: 2258-014X

Editor

EuroPhilosophie Editions

Referencia electrónica

Mònica Carbó Ribugent, « Juicio y ser. Kant y Fichte en la encrucijada hacia el romanticismo », Revista de Estud(i)os sobre Fichte [En línea], 6 | 2013, Publicado el 03 junio 2013, consultado el 10 diciembre 2020. URL : http://journals.openedition.org/ref/425 ; DOI : https://doi.org/10.4000/ref.425

Este documento fue generado automáticamente el 10 diciembre 2020.

(c) EuroPhilosophie 


\title{
Juicio y ser. Kant y Fichte en la encrucijada hacia el romanticismo
}

\author{
Mònica Carbó Ribugent
}

1 El debate historiográfico sobre el papel de Hölderlin en el desarrollo del idealismo alemán surgido en las últimas décadas ha arrojado luz sobre el complejo entramado de relaciones e influencias recíprocas entre las propuestas filosóficas no sólo de las figuras más destacadas del escenario intelectual entre Kant y Hegel, sino también de otros autores que de modo más o menos explícito reclaman ser incorporados a dicho debate. ${ }^{1}$

2 A partir de la lectura de la correspondencia de Hölderlin se puede afirmar por lo menos que su afición a la filosofía es paralela y contemporánea a su afición por la poesía: las referencias a su propia producción poética se intercalan con referencias a sus máximos intereses intelectuales: Kant y los griegos, pero también Fichte y Spinoza. Para el propósito de nuestro trabajo no es irrelevante la cuestión de la clasificación de Hölderlin como filósofo del idealismo o como poeta romántico sui-generis. Basta con constatar el camino poético que va a seguir Hölderlin después de su modesta, aunque fulgurante, intervención en el núcleo mismo de gestación del proyecto idealista para darse cuenta de que el personaje rehúye cualquier intento de clasificación estandarizada.

3 La ausencia de un cuerpo articulado de reflexiones filosóficas en la obra de Hölderlin dificulta la interpretación de su pensamiento. Tal tarea exige ciertamente continuar dicha interpretación al filo de su producción poética más allá de su breve aportación crítica, cifrada casi de forma exclusiva en el fragmento Urtheil und Seyn $(U u S)^{2}$ de 1795 , a la cuestión central de la filosofía en los años convulsos de la recepción del kantismo en Jena. ${ }^{3}$

4 Sin embargo, en el marco del presente trabajo intentaré solamente mostrar que el fragmento UuS, que contiene de forma germinal la filosofía de Hölderlin articulada como crítica al fundamento del idealismo transcendental presentado por Fichte en su $G W L^{4}$, revela la crítica de Hölderlin a la apropiación idealista de la filosofía kantiana en la pluma de Fichte. ${ }^{5}$ 
Un primer contacto con el texto de Hölderlin nos permitirá detectar ciertos giros de clara inspiración fichteana. Hölderlin utiliza términos e expresiones de cuño fichteano tales como: 'intuición intelectual', 'relación recíproca entre sujeto y objeto', 'Yo soy yo', 'autoconsciencia' e 'identidad'. No queda duda entonces de la filiación netamente fichteana del vocabulario filosófico de Hölderlin en UuS. De modo que el fragmento documenta no solamente el interés de Hölderlin por el problema de la fundamentación de la experiencia -central en el debate filosófico del momento-, sino también la extraordinaria capacidad de penetración en el problema que exhibe Hölderlin en su crítica rigurosa a la manera en que Fichte pretende haber resuelto dicha cuestión.

Centrémonos para empezar en el primer fragmento, que lleva como título el término "Juicio". Juicio significa, según Hölderlin, "separación originaria entre objeto y sujeto"6. Debemos poner atención en el hecho de que Hölderlin inicie su análisis a partir de una separación. Fichte había partido en su GWL, por el contrario, del análisis de aquello en que la experiencia se nos presenta unido (sujeto y objeto en la autoconsciencia individual), ${ }^{7}$ a pesar de que la unidad se muestre en la reflexión en su escisión. ${ }^{8}$ Esta escisión sin embargo no es para Fichte un hecho originario del Yo. La reflexión encuentra los opuestos ya unidos y no como "producto de la reflexión", sino como "su descubrimiento" (Fund). ${ }^{9}$ Para Hölderlin, por el contrario, la experiencia, el juicio, es la separación entre sujeto y objeto. El juicio tal como lo entiende Hölderlin dice, pues, mucho más, ya que él es la separación por la cual, y solo por la cual, sujeto y objeto son posibles. De allí que dicha separación que sucede en el juicio es "partición originaria" (Ur-Theilung).

7 Por ser separación, empero, muestra la necesidad de una unidad previa como presuposición necesaria de la dualidad sujeto-objeto. Hölderlin identifica esta unidad previa con la intuición intelectual en lo que acaba por ser una reinterpretación de la misma figura en la filosofía de Fichte. ${ }^{10}$

8 Avanzando en UuS podemos leer que según Hölderlin la expresión fichteana 'Yo soy Yo' expresa en realidad la partición y no, como Fichte sostiene, la unidad de sujeto y objeto. Según Hölderlin este juicio es el ejemplo más pertinente de la partición originaria teórica, es decir, aquella en que el segundo Yo, es en realidad "el No-Yo" en sentido de objeto teórico. ${ }^{11}$ Este juicio, sin embargo, no parece ser tan pertinente para expresar la partición originaria práctica, en la cual la oposición o separación se produce entre el primer Yo y otros 'Yo'. ${ }^{12}$

Nos parece que lo dicho hasta aquí obliga a matizar con prudencia la innegable huella de Fichte. Hölderlin dedica el primer parágrafo de su texto, "Juicio", al desarrollo de la partición originaria de un todo en sujeto y objeto. Identifica el todo con la intuición intelectual, y sin embargo la expresión de la identidad 'Yo soy yo' se señala como ejemplo de la partición originaria. Cierto es que a Hölderlin no se le escapa que este ejemplo de partición expresa en efecto también una unión -a través de la cópula-, o sea, una identidad. Sin embargo esta identidad pertenece a un orden ontológico 
distinto del de la partición originaria. Por ello procede en la segunda parte del texto, "Ser", a diferenciar entre modos de ser. sujeto y objeto. Mas dicho vínculo no tiene siempre la misma intensidad. En el caso de la intuición intelectual, sigue Hölderlin, sujeto y objeto están absolutamente unidos en una forma tal que no cabe proceder jamás a su separación. Sujeto y objeto se hallan en la intuición intelectual tan unidos que no podemos verlos como sujeto y objeto sino tan sólo como unidad previa a cualquier separación entre sujeto y objeto. Tan unidos que son lo absolutamente uno, el "Ser Absoluto". El tipo de ser o unión que aparece en cambio en la identidad "Yo soy Yo" no es para nada absoluto. Es, según Hölderlin, una unión secundaria o mediada. Ello queda probado, dice Hölderlin, en lo siguiente: no es el caso que no podamos proceder a una separación. En realidad, nos hallamos ante una separación, pues la identidad es sólo posible gracias a la separación que tiene lugar en la autoconsciencia, en la cual Yo me aparezco como objeto al separarme y reconocerme ante aquello que aparece ante mí.

11 Hölderlin puntualiza en ambos casos que la prueba consiste en poder proceder a la separación sin que el hacerlo suponga vulnerar o desnaturalizar aquello que pretendemos separar. Por ello, en el caso de la intuición intelectual parece claro que en el momento en que intentemos separar aquello que se da como íntimamente unido, estaremos pervirtiendo el carácter absoluto de esa unión. Contrariamente, en el caso de la identidad, como hemos visto, tal cosa no sucede, porque la unión no es inmediata, sino mediada por la consciencia. De modo que aquello que nos disponemos a aislar o separar (sc. el Yo) es únicamente posible a través de la separación que tiene lugar en la autoconsciencia. intelectual también la encontramos en la filosofía de Fichte, para quien el Yo como autoconsciencia es un producto del actuar originario, es decir: para que aparezca el Yo, el actuar debe revertir en sí mismo, poderse ver reflejado en su propio producto. ${ }^{14}$ Hölderlin coincide con el tratamiento que da Fichte a la figura de la autoconsciencia. Pero para Fichte la operación de separación es solamente condición para tomar consciencia de la unidad, de la identidad absoluta entre sujeto y objeto que está en la base de todo lo que ocurre en la consciencia. ${ }^{15}$ Para Fichte el Yo en cuanto Thathandlung es previo a y condiciona toda la serie de actos o hechos (Tatsachen) de la consciencia, de allí que deba postularse su carácter incondicionado y absoluto. ${ }^{16}$ Sería intolerable para Fichte que el Yo en cuanto actuar libre pudiera estar condicionado por algo externo a sí mismo. Él mismo es condicionante y por ende constitutivo de toda realidad para el Yo. Hölderlin, atento a las lecciones del maestro, moviéndose en sus mismos parámetros, hablando de la misma operación (autoconsciencia) denuncia aquí el error de considerar como unidad absoluta algo que es fundamentalmente una partición. Algo que es también un cierto tipo de unidad, pero de ningún modo una unidad absoluta, sino antes bien una unidad mediada por una partición. Este es el sentido, según mi lectura, de la frase con la que el fragmento concluye: "La identidad no es = el Ser absoluto" ${ }^{17}$.

Resumiendo: Hölderlin sostiene siguiendo a Fichte que en la intuición intelectual hallamos una unidad absoluta de sujeto y objeto, lo que Hölderlin llama "ser sin más" 
(Seyn schlechthin). Dicha intuición intelectual, sin embargo, no se expresa en la forma de la identidad o 'Yo soy Yo'. La identidad es unión de sujeto y objeto a través de la consciencia, es decir, mediada por la separación entre sujeto y objeto. En otras palabras, para Hölderlin la autoconsciencia no coincide con la intuición intelectual. Aun así Fichte y Hölderlin comparten la idea de que la autoconsciencia es el fundamento de toda experiencia. Hölderlin admite que el Juicio o partición originaria es la separación gracias a la cual sujeto y objeto son posibles y reales. En otras palabras Hölderlin acepta la autoconsciencia como condición de posibilidad de la experiencia, pero no le otorga el carácter de fundamento absoluto. Mientras que Fichte había declarado que la autoconciencia en cuanto Thathandlung era el fundamento absoluto de la experiencia. ${ }^{18}$

De acuerdo con lo dicho hasta aquí, UuS articula una crítica general a la filosofía de la consciencia según la cual el ser absoluto jamás podrá ser transparente a la consciencia, porque entonces dejaría de ser absoluto. La conclusión más importante es que ni el saber ni la experiencia podrán ser absolutos, pues la pretendida unidad que Fichte había promulgado entre ser y saber ha quedado, según Hölderlin, refutada. La crítica al absolutismo de la consciencia supone por ello una relativización del estatuto del saber: toda relación entre sujeto y objeto es una unión mediada que se expresa en la forma del juicio. Pero el ser absoluto o unión inmediata de sujeto y objeto, siempre previa, es una presuposición necesaria del juicio: "En el concepto de partición ya se encuentra el concepto de la relación recíproca entre Objeto y Sujeto y la presuposición necesaria de un Todo del cual Objeto y Sujeto son las partes' (sic) ${ }^{19}$. Por todo ello, el ser sin más no es accesible a la conciencia, aunque ciertamente es solamente pensable gracias a la autoconsciencia. Se sigue pues, que aun siendo algo previo $a$, no es empero indiferente $a$ la autoconciencia. Así descubrimos la paradoja, que nos lleva a cierta perplejidad, de remitir a algo que está más allá de nuestra consciencia pero que sólo aparece en su escindirse en nuestra consciencia.

Nada más lejos de la intención de Hölderlin que renunciar a su incansable búsqueda de lo absoluto. El ser absoluto nos hace falta para comprender la escisión en sujeto y objeto. Pero habrá que buscarlo más allá de la consciencia. Dado el carácter escurridizo de este fundamento presentado en el fragmento que nos ocupa habrá que aceptar con Hölderlin que el ser absoluto no será jamás objeto de ningún saber finito. Es solamente una presuposición necesaria del juicio en cuanto que Ur-theilung y por ello es el saber que todo saber es necesariamente limitado.

El manuscrito de Hölderlin ofrece sin embargo un nuevo desarrollo de la cuestión utilizando en este caso terminología kantiana. El análisis ocupa el segundo parágrafo del texto que Hölderlin dedica al juicio, en cuyos últimos puntos son ahora convocadas tres facultades de la razón, a saber: razón, entendimiento e intuición. A cada facultad le corresponde una categoría modal: necesidad, posibilidad, realidad, respectivamente. ${ }^{20}$

Veamos cómo opera esta atribución de modos a las facultades, y también en qué sentido se interpreta cada facultad. El parágrafo se inicia afirmando los paralelismos realidad/ posibilidad y conciencia mediada/conciencia inmediata. El sentido de ambos paralelismos parece cambiar de la primera proposición a la siguiente: en la primera parece que la realidad correspondería a la conciencia mediada y la posibilidad a la conciencia inmediata. Pero a continuación Hölderlin afirma que un objeto es pensado 
como posible cuando "se repite la conciencia anterior en virtud de la cual el objeto es real". Según esta última proposición, pues, la inmediatez, queda adjudicada al carácter fenoménico de la intuición y a la realidad. Ello se vería confirmado por las ulteriores afirmaciones del parágrafo y también por el sentido global que pueda suponer este análisis en terminología kantiana del fragmento. Si aceptamos esta breve consideración podemos constatar que nos hallamos ante una facultad de percepción, una consciencia inmediata de la experiencia como fenómeno, que tratamos en términos de realidad (inmediatez). Dicha experiencia, a través de la consciencia mediada o pensamiento se nos hace objeto, y la tratamos en términos de posibilidad (constitución). Hölderlin sostiene entonces que todo lo que forma parte de la consciencia mediada, proviene de la consciencia inmediata, de manera que sólo podemos pensar como posible aquello que antes fue real en la intuición. Parecemos estar inmersos en el análisis kantiano de las facultades cognoscitivas, concretamente en la relación entre intuición y entendimiento. El conocimiento como síntesis de intuición (realidad) y concepto (posibilidad). ${ }^{21}$ Hölderlin insiste en el hecho que sólo podemos conocer aquello que forma parte de la experiencia posible, puesto que el objetivo de este análisis es la contraposición de las categorías de realidad y posibilidad -y por ello de las facultades de Intuición y Entendimiento- frente a la categoría necesidad -y por ello de la Facultad de la Razón.

Hölderlin concluye que en la medida en que las representaciones de la razón (sc. ideas) no son accesibles a la consciencia (en sentido idealista), es decir, jamás podremos tratarlas como objetos de conocimiento, y por lo mismo, tampoco como intuiciones, a dichas representaciones les corresponde la categoría de necesidad. Podemos entender en este contexto el carácter de necesidad como inevitabilidad, para constatar así la sintonía entre la conclusión de este análisis à la Kant y la del anterior análisis à la Fichte. La conclusión consiste en subrayar la oposición entre conocimiento, saber y conciencia por un lado, y ser absoluto por otro. En términos kantianos Hölderlin sostiene que el conocimiento como síntesis de intuición y concepto es un conocimiento limitado al fenómeno. Sólo la razón mediante ideas puede proporcionarnos una aproximación a lo absoluto y a la totalidad. En términos fichteanos Hölderlin había sostenido que la autoconsciencia como unión de sujeto y objeto es una figura limitada y por ende incapaz de fundamentar el saber absoluto. Lo absoluto queda siempre más allá de la consciencia y el saber finito. Por lo tanto, el saber de la autoconsciencia no ofrece una herramienta adecuada para la aproximación a lo absoluto.

Así las cosas hay que admitir que el análisis en términos kantianos no ofrece novedades significativas. Sin embargo la introducción de la categoría de necesidad facilita el tránsito de un registro (el de la limitación del juicio) a otro (el carácter absoluto del ser). Lo que este segundo análisis suscita, es la sospecha de que mientras la denuncia a Fichte parece explícita, no nos parece que Hölderlin se muestre crítico ante el planteamiento kantiano.

Detengámonos en primer lugar a observar más de cerca las relaciones entre Fichte y Kant por lo que se refiere al problema de lo absoluto, que es precisamente el tema del que se ocupa el fragmento. La interpretación fichteana del kantismo nos la ofrece el propio Fichte en su ZE. ${ }^{22}$ Recordemos como ahí Fichte se presenta a sí mismo el auténtico continuador del kantismo, frente a la lectura dogmática de Kant. Su Doctrina 
de la Ciencia, nos dice, no es otra cosa que filosofía kantiana bien comprendida. La intención de Fichte es demostrar que su concepción filosófica estaría ya implícitamente formulada en Kant. Para ello deberá superar los reproches del kantismo dogmático, cuya interpretación es radicalmente incompatible con el idealismo absoluto. Es sabido que el núcleo de la polémica entre los lectores dogmáticos de Kant y el propio Fichte consiste en el status de la cosa-en-sí. Fichte creía haber liquidado la polémica afirmando que la cosa-en-sí, en último término era algo añadido por nuestro propio pensamiento, y por ende, de ningún modo una objetividad que pueda determinarnos. Según Fichte, además dicha solución se hallaría implícita en la propia obra de Kant, puesto que aplicar una categoría más allá del campo del fenómeno supondría un grave error también en términos kantianos: suponer una cosa-en-si afectándonos desde fuera sería sólo posible aplicando la categoría de causalidad desde aquello fundamentado (objeto) al fundamento (sujeto), lo cual, según Fichte, es ilegítimo ya desde una perspectiva kantiana. Por esta razón Fichte cree justificado identificar su propio idealismo con el idealismo transcendental de Kant. Fichte añade también que el Yo puro, principio absoluto del idealismo, coincide con la figura kantiana de la apercepción pura: el Yo que aparece como fruto de la reflexión del concepto en un sujeto que piensa (es decir como subjetividad simultánea a la objetividad), y que según Kant debe acompañar todas mis representaciones para que haya unidad en el conocimiento (el conocimiento debe ser compatible para uno y el mismo sujeto), es según Fichte el Yo puro, tal como viene presentado en la Doctrina de la ciencia. Esta identificación implica conceder carácter determinado a toda experiencia (cualquier otra consciencia no pura) de dicho Yo o consciencia de sí. Fichte admite con honestidad que Kant se había limitado a constatar el carácter condicionante del Yo pienso o apercepción pura, pero se muestra convencido de que si bien no se demuestra en Kant que dicha consciencia de sí determina (es decir, constituye fundamento de) toda experiencia, hay que suponer que Kant habría pensado en estos términos, porque sólo así, según Fichte, cobran sentido la mayoría de sus afirmaciones.

21 Fichte se atreve incluso a indicar el lugar donde Kant debería haber situado la consideración del Yo puro como principio del sistema: en la medida en que para Fichte el Yo puro es un mero actuar y no un ser, el recurso al imperativo categórico es irresistible. Frente a él se pregunta: ¿qué tipo de conciencia es el imperativo categórico sino una pura conciencia inmediata de sí como mero actuar, es decir aquello que Fichte entiende como Yo puro o intuición intelectual? Vemos como paso a paso, la lectura fichteana de Kant se despliega como una reducción progresiva de toda dualidad, o como sometimiento del objeto al sujeto, del conocer al actuar, con la mirada puesta en hallar un principio que lo contenga todo, y reducir toda experiencia a un desarrollo de dicho principio. Descubrimos así el fundamento absoluto en nosotros. Nosotros somos lo absoluto. Y Fichte sostiene que dicha tesis está latente en Kant.

La prudencia de Fichte al matizar que dicha tesis está sólo latente abre la posibilidad a otra interpretación de Kant. Ello cobra importancia especialmente en el contexto del fragmento Urtheil und Seyn. Parece que en la argumentación de Hölderlin lo que está en juego es principalmente el anhelo de sistema, y por ello la necesidad de un fundamento absoluto en el sujeto. Lo cual explicaría la incomodidad de este discípulo de Kant ante la incapacidad del maestro para desarrollar un sistema, el fundamento del cual habría 
quedado tan sólo indicado en la $\mathrm{KrV}$, aunque sobre él jamás se habría levantado el sistema de la filosofía. Como consecuencia en la filosofía de Kant el sujeto goza de un conocimiento radicalmente cierto, pero necesariamente finito. El establecimiento de los límites del conocimiento excluye necesariamente un gran número de representaciones y aspectos de la vida del hombre. Para Fichte, en cuanto que pensables, dichos aspectos y representaciones deben ser incluidos en la serie de la consciencia, y por lo tanto, en última instancia todo quedaría fundamentado en el principio absoluto de la autoconsciencia en cuanto Thathandlung, en el sí del cual se generarían como necesarios todo y cada uno de sus momentos.

Volviendo al texto de Hölderlin deberemos constatar que la crítica de Hölderlin a Fichte tiene su núcleo precisamente en aquellos movimientos con que Fichte lanza su interpretación particular de Kant. Al filo de lo que hemos leído en la sección del fragmento dedicada a las formas de la modalidad, todo parece indicar que Hölderlin respeta el carácter finito del conocimiento en Kant así como la contraposición radical entre todo aquello que pertenece al conocimiento (aquello que tiene lugar en la consciencia) y todo aquello que se excluye del conocimiento (ideas).

La radicalidad de la crítica de Hölderlin se hace algo más patente cuando se extiende hacia los acercamientos, pretendidamente respetuosos, al carácter pre-judicativo de lo absoluto. Pero esta conclusión no aparece en el fragmento en cuestión. El desarrollo particular de Hölderlin de la asunción consecuente del axioma de la exclusividad de la belleza para la manifestación de la unidad-totalidad puede analizarse en la novela Hyperión (1795) -su primera obra publicada- en la que podemos detectar la proyección de problemas filosóficos propios del idealismo incipiente. Un análisis detallado de la novela nos permite valorar hasta qué punto puede ser considerada como la formulación poética de un problema filosófico.

Si el fundamento no puede ser accesible a la conciencia, porque ella es siempre una unidad parcial de Sujeto y objeto, una unidad de relaciones, ¿cómo podemos experimentar en algún modo este fundamento? Habrá que recurrir a algún tipo de presentación no discursiva. Efectivamente, Hölderlin apunta a un absolutamente primero que por su propia naturaleza escapa siempre al discurso, al juicio (la escisión es la pérdida de este Seyn, pero al Seyn le es inherente el perderse). En términos kantianos, podríamos decir que se apunta a algo que está más allá de toda validez teórica objetiva. Tal vez aquello que aparece en la tercera crítica kantiana como lo bello. Sabemos que Hölderlin fue un ávido lector de esta obra, aunque no podamos probar hasta qué punto la evolución de su pensamiento siguió esta vía de argumentación ${ }^{23}$ lo que sí es constatable es que en el año de redacción de Urtheil und Seyn (1795) Hölderlin está también trabajando en su novela lírica Hyperion

Para nuestro interés, lo más destacable de la novela es el sentimiento de exclusión paralelo a la justa reivindicación del Uno-todo (-hen kai pan-) que toma en boca de Hyperión un tono desesperado, los distintos intentos de acercamiento al cual son vividos como consuelo momentáneo. El máximo consuelo viene proporcionado intermitentemente a lo largo de la novela por lo que podríamos definir cómo una fusión con la naturaleza en cuanto que espacio de divinidad y a la vez patria del hombre. El concepto de naturaleza en Hyperión funde, por lo tanto, el sentido del panteísmo 
spinozista $^{24}$ con el de aquella unidad absoluta de sujeto y objeto schellingiana según la cual el sujeto no es más que una forma sofisticada de la natura naturans. La naturaleza sentida como Uno-todo se halla a la base del sentimiento simultáneo de exaltación y serenidad: por ser a la vez aquello más sublime y elevado y también aquello en que, siendo el hogar del hombre, reposamos con absoluta naturalidad. Pero: Hyperión solo alcanza esta plenitud en instantes aislados viéndose siempre nuevamente arrojado a su condición escindida. Ello lo impulsa a perseverar en la búsqueda.

Este proyecto de recuperación de la unidad, o de reunificación, proyecto que se vuelve característico en el romanticismo alemán, pierde en Hyperion la ingenuidad platónica, de modo que la reunificación efectiva queda descartada y el acento se carga en la finitud radical de la formulación platónica de vivir o pensar la unidad como recuerdo en la carencia. La indigencia es necesaria para poder al menos sentir aquella unidad como recuerdo. Del mismo modo en que de acuerdo con UuS sujeto y objeto tenían que escindirse en la consciencia para que su unidad previa apareciera en el horizonte de la partición. La unidad del ser no es nunca resultado, más bien siempre tarea eterna del pensamiento. Tarea que a su vez se revela como necesariamente infructuosa puesto que la reconciliación es solo posible a través de la escisión.

Esta contradicción puede ser la clave para establecer el vínculo entre el proyecto meramente especulativo de Urtheil und Seyn y el proyecto literario de Hyperion: una tal contradicción, empero, no puede formar parte de un sistema filosófico, sostiene Hölderlin. Sólo la poesía, según él, puede vehicularla. Para ser fiel a sus objetivos, la poesía se reivindica ahora como meta-discurso, como única herramienta capaz de plasmar lo más alto, lo sagrado (la unidad del ser como Uno-todo). Así, la belleza quedará situada en el claroscuro de la presencia-ausencia de la unidad, cómo única experiencia legítima, en cuanto que permite reflejar el carácter enigmático de una unidad que se muestra sólo en su pérdida.

A pesar del tono cuasi-romántico del pensamiento de Hölderlin, no creo que el desplazamiento de Hölderlin hacia el tratamiento poético del problema filosófico pueda identificarse sin más con la estrategia de la lectura romántica del idealismo. En primer lugar, porque lo que según nuestra interpretación hallamos en el fragmento Juicio y Ser es sin lugar a dudas una crítica radical al planteamiento del absolutismo de la consciencia idealista. Y aunque este ataque frontal lanzado por Hölderlin ha sido a menudo juzgado por algunos como comprometido con la búsqueda genuinamente idealista de un absoluto, ${ }^{25}$ también se ha defendido la posible inspiración kantiana de dicha crítica. ${ }^{26}$ En segundo lugar, y apoyándome en esta última interpretación, no me parece que Hyperión como proyecto literario encaje con la intención romántica de una apropiación estética de lo absoluto, sino que más bien es la constatación reiterada de la imposibilidad de una apropiación de lo absoluto.

\section{Bibliografía}

Acosta, E., "Vier Bestimmungen des Nicht-Ich in der Jenenser Periode der Fichteschen Wissenschaftslehre", en: Pfeiffer, M. \& Rapic, S. (eds.) Das Selbst und sein Anderes : Festschrift für Klaus Erich Kaehler, München: Alber, 2009, pp.98-108. 
Fichte, J.G., J.G. Fichte-Gesamtausgabe der bayerischen Akademie der Wissenschaften, Stuttgart-Bad Cannstat: Fromman Verlag, 1962

Frank, M., "Unendliche Annäherung". Die Anfänge der philosophischen Frühromantik, Frankfurt a. M.: Suhrkamp 1997

Auswege aus dem deutschen Idealismus, Frankfurt a. M.: Suhrkamp 2007.

Henrich, D., Grundlegung aus dem Ich. Untersuchungen aus der Vorgeschichte des Idealismus. Tübingen-Jena, 1790-94, Frankfurt a.M.: Suhrkamp 2004.

Der Grund im Bewusstsein. Untersuchungen zu Hölderlins Denken (1794-1795).

Stuttgart: Klett-Cotta 1992.

Konstellationen. Probleme und Debatten am Ursprung der idealistischen Philosophie. Stuttgart: Klett-Cotta 1991.

Hölderlin, F., Sämtliche Werke, Grosse Stuttgarter Ausgabe, Friedrich Beissner, Stuttgart: W.Kohlhammer Verlag, 1943

Jamme, Ch., "La evolución filosófica de Hölderlin", en Sileno, Hölderlin, pp. 32-42, Madrid, 1999.

Janke, W., Transzendentalphilosophie als System. Die Auseinandersetzung zwischen 1794 und 1806. Hamburg: Felix Meiner, 1989.

Kant, I., Immanuel Kant Werke in Sechs Bänden, Wilhelm Weischedel, Darmstadt: Wissenschaftliche Buchgesellschaft, 1957

Martínez M., F. De Kant a Hölderlin, Madrid: Visor, 1992. Hölderlin y la lógica hegeliana, Madrid: Visor, 1995.

"La «Crítica del Juicio» y la cuestión Grecia-Modernidad", en AA. VV. Estudios sobre la Crítica del Juicio, Madrid: Visor y C.S.I.C., 1990, pp. 147-164.

Serrano, V. Absoluto y conciencia. Una introducción a Schelling, Madrid: Plaza y Valdés, 2008

Taminiaux, J.J. La nostalgie de la Grece a l'aube de l'Idealisme Allemand. Kant et les grecs dans les itineraires de Hegel, Schiller et Hölderlin, La Haye: Nijhof, 1967

Wegenast, M. Hölderlins Spinoza-Rezeption und ihre Bedeutung für die Konzeption des ‘Hyperion'. Tübingen: Max Niemeyer,1990

\section{NOTAS}

1. Véase: Frank 1997 y 2007; Henrich 1991, 1992 y 2004; Janke 1989; Martínez Marzoa 1995.

2. Hölderlin, F., Urtheil und Seyn, en: Werke-Stuttgarter Ausgabe (StA), t. 4, pp. 216/217.

3. Si bien V. Serrano sostiene que el fragmento debería ser leído como un desarrollo de planteamientos aparecidos anteriormente en algún ensayo y en la correspondencia entre Hegel y Hölderlin (2008: 82). Jamme, por su parte, advierte la insuficiencia de la referencia a la filosofía de Fichte para comprender bien el fragmento (1999: 32- 42).

4. En el momento de redacción del manuscrito Urtheil und Seyn Fichte había ya pronunciado sus lecciones en Jena, y a partir de la correspondencia completa de Hölderlin se puede comprobar también su efectiva asistencia a las sesiones de Fichte. 
5. Aunque, según Martínez Marzoa (1995) Hölderlin no podría ser consciente de dicha conexión pues su conocimiento de Kant en este momento estaba aún mediado por la propia interpretación de Fichte.

6. Cf. StA, 4, p.216

7. Cf. GWL, GA I/2, 256, 264, 284 y 287.

8. Cf. GWL, GA I/2, 284.

9. Ibíd.

10. Ciertamente el concepto de intuición intelectual no se halla en GWL, pero sí en otros escritos del período de Jena de Fichte como la Segunda introducción a la doctrina de la ciencia de 1797. (ZE, GA I/4, 225) Véase además WLnm-K, GA IV/3, 347.

11. Acerca de la pluralidad de significados que adquiere el término No-Yo en la filosofía de Fichte véase Acosta 2009.

12. Cf. StA, 4, p.216.

13. Cf.StA, 4, p.217.

14. Cf. GNR, GA I/3, 313-318 y VND, GA I/4, 271-274.

15. Cf. GWL, GA I/2, 284.

16. Cf. GWL, GA I/2, 255.

17. StA, 4, p. 217.

18. Cf. GWL, GA I/2, 255

19. Cf. StA, 4, p. 216

20. Cf. StA, 4, p.217

21. $K r V$ B130-168.

22. Me refiero al conocido $\S 6$ de ZE, GA I/4, 221-244. En lo que sigue nos ceñimos exclusivamente a este parágrafo.

23. Véase al respecto Martínez Marzoa 1995 y 1990: 147-164 ; y Taminiaux 1967.

24. Véase Wegenast 1990

25. Como defiende sobretodo Henrich, op.cit

26. Sería el caso de F.M. Marzoa, op.cit, y en cierto modo también de J.J. Taminiaux, op.cit

\section{RESÚMENES}

It is known that the German poet Friedrich Hölderlin was a passionate reader of Kant. There is evidence of his parallel interest on poetry and philosophy in his personal correspondence, where references to his own poetical production intertwine with references to his main intellectual interests: the study of Kant and the Greeks. Hölderlin, however, was a colleague of Hegel and Schelling during the years in Tübingen, and he attained personally Fichte's lessons. His peculiar position on the philosophical debate between Kant and Idealism is thoroughly expressed in the manuscript Urtheil und Seyn from 1795. A careful reading of the text provides an account of the tension between Kant's philosophy and Fichte's idealistic approach. Hölderlin displays a critical argument against Fichte's denial of the original unity expressed in the Kantian formula of an "unknown common root". Such a pre-reflexive unity is discarded by Fichte as being unable to bear fruit, and in its place he posits an absolute principle, the identity of the self, the absolute I. Hölderlin indicates the incoherence of stating as "absolute" something which is reflexive, and therefore a result of a division, thus casting a straightforward attack to the nuclear ground of the 
idealistic project. Such an attack can ultimately be interpreted either as a sound critique to idealism main principle, but still engaged with the idealistic privileged search of an absolute ground or as a sheer opposition to Idealism -in as much as Hölderlin's critique seems to emerge out of respect for the Kantian position. However, Hölderlin's further development of this issue in literary form does not provide an argument to identify his approach to the absolute with that of the romantics.

\section{ÍNDICE}

Keywords: Hölderlin, Fichte, Kant, (theory of) judgement, idealism, romanticism

\section{AUTOR}

MÒNICA CARBÓ RIBUGENT

Universitat de Girona 